\title{
Peer Coaches to Improve Diabetes Outcomes in Rural Alabama: A Cluster Randomized Trial
}

Monika M. Safford, $M D^{1}$

Susan Andreae, MPH

Andrea L. Cherrington, MD, MPH

Micbelle Y. Martin, $P b D^{1}$

Jewell Halanych, MD, MSc ${ }^{1}$

Marquita Lewis, MS

Ashruta Patel, MSc ${ }^{1}$

Ethel Jobnson ${ }^{1,2}$

Debra Clark, $\mathrm{MS}^{1,3}$

Cbristopber Gamboa, MS

Josbua S. Richman, MD, $P b D^{4,5}$

'Department of Medicine, School of Medicine, University of Alabama at Birmingham, Birmingham, Alabama

${ }^{2}$ West Central Alabama Community Health Improvement League, Camden, Alabama

${ }^{3}$ Sumter County Health and Wellness Education Center, Livingston, Alabama

${ }^{4}$ Birmingham VA Medical Center, Birmingham, Alabama

${ }^{5}$ Department of Surgery, University of Alabama at Birmingham and the Birmingham Veterans Affairs Medical Center, Birmingham, Alabama

Conflicts of interest: authors report none.

\section{CORRESPONDING AUTHOR}

Monika M. Safford, MD

1717 11th Avenue South

Birmingham, AL 35294-4410

msafford@uab.edu

\begin{abstract}
PURPOSE It is unclear whether peer coaching is effective in minority populations living with diabetes in hard-to-reach, under-resourced areas such as the rural South. We examined the effect of an innovative peer-coaching intervention plus brief education vs brief education alone on diabetes outcomes.
\end{abstract}

METHODS This was a community-engaged, cluster-randomized, controlled trial with primary care practices and their surrounding communities serving as clusters. The trial enrolled 424 participants, with 360 completing baseline and follow-up data collection (84.9\% retention). The primary outcomes were change in glycated hemoglobin $\left(\mathrm{HbA}_{1 \mathrm{c}}\right)$, systolic blood pressure (BP), low density lipoprotein cholesterol (LDL-C), body mass index (BMI), and quality of life, with diabetes distress and patient activation as secondary outcomes. Peer coaches were trained for 2 days in community settings; the training emphasized motivational interviewing skills, diabetes basics, and goal setting. All participants received a 1-hour diabetes education class and a personalized diabetes report card at baseline. Intervention arm participants were also paired with peer coaches; the protocol called for telephone interactions weekly for the first 8 weeks, then monthly for a total of 10 months.

RESULTS Due to real-world constraints, follow-up was protracted, and intervention effects varied over time. The analysis that included the $68 \%$ of participants followed up by 15 months showed only a significant increase in patient activation in the intervention group. The analysis that included all participants who eventually completed follow-up revealed that intervention arm participants had significant differences in changes in systolic BP $(P=.047)$, BMI $(P=.02)$, quality of life $(P=.003)$, diabetes distress $(P=.004)$, and patient activation $(P=.03)$, but not in $\mathrm{HbA}_{1 \mathrm{c}}(P=.14)$ or LDL-C $(P=.97)$.

CONCLUSION Telephone-delivered peer coaching holds promise to improve health for individuals with diabetes living in under-resourced areas.

Ann Fam Med 2015;13(Suppl_1):S18-S26. doi: 10.1370/afm.1798.

\section{INTRODUCTION}

7 he Southeast has both the highest prevalence of diabetes and the highest stroke and heart disease mortality in the United States.

1 Risks of poor outcomes can be reduced by controlling blood glucose, blood pressure (BP), cholesterol levels and body weight, but accomplishing this is difficult. In rural areas like the Alabama Black Belt, ${ }^{2}$ barriers to achieving risk factor control are particularly daunting: one-third of area residents live below the federal poverty line (compared with 15\% nationally $)^{3,4}$ and there are fewer than half as many primary care physicians per 10,000 population as the US average. ${ }^{3,5,6}$ Low educational attainment is common $^{7}$; the area's residents are predominantly African Americans, and mistrust of the health care system is widespread. ${ }^{8}$ Low-cost programs that can overcome these barriers are urgently needed.

Peer-support interventions to promote self care are particularly attractive for such under-resourced regions. Peer coaches are typically lay people who receive minimal training. They live in the same communities 
as the targeted population and therefore have in-depth understanding of the challenges of carrying out physicians' self-care recommendations. Peer coaches have been shown to improve asthma, cancer screening and treatment, and diabetes outcomes among low-income minorities. ${ }^{9-16}$ It is not known, however, whether peer coaches can be effective in rural, under-resourced settings, where distance barriers are considerable and telephone connections are intermittent, or whether peer coaches are superior to diabetes education alone, especially in areas where basic knowledge about diabetes and healthy lifestyles is low. To fill these gaps in the evidence, we conducted a cluster-randomized trial in a rural, under-resourced region that tested the effectiveness of peer coaches plus brief education compared with brief education alone.

\section{METHODS}

\section{Settings and participants}

Our partnering communities, each served by at least one primary care practice, were located in 8 counties of the Alabama Black Belt. Details of engagement, recruitment and intervention development are reported elsewhere. ${ }^{17-19}$ Briefly, adults who had been told by a doctor or nurse they had diabetes and who wanted help with self-management were eligible to participate. Individuals were excluded from participation if they did not have a primary care provider, had advanced illness with limited life expectancy, planned to move out of the area within the next year, or were unwilling to work with a peer coach over the telephone. Participants were recruited using respondentdriven sampling, ${ }^{20}$ a method designed to engage hardly reached populations. All participants provided written informed consent. The University of Alabama at Birmingham Institutional Review Board approved the study protocol. Data collection details are provided in the Supplemental Appendix at http://www. annfammed.org/content/13/Suppl_1/S18/suppl/DC1.

\section{Peer coach training}

Peer coaches were recruited from the same communities as participants, and all provided written informed consent. Peer coaches had to have diabetes themselves or to care for someone in their family with diabetes. Peer coaches completed 12 hours of training over 2 days, covering the basics of diabetes, healthy eating, physical activity, motivational interviewing, community resources, ethics of research, and the study protocol. Peer coaches were taught how to develop realistic action plans and help participants work toward their self-selected goals, providing social and emotional support and helping participants access support from oth- ers. They were also taught how to make the most of doctor visits. Of the 68 candidates who were trained, 41 were successfully certified to become peer coaches and went on to be paired with 2-14 (mean 6-7) intervention participants within the same community.

\section{Coaching sessions}

Each coach-participant dyad met for 45-60 minutes in person or over the telephone to get to know each other, go over the participant's personalized diabetes report card, and select a personal goal. Peer coaches telephoned participants weekly for the first 2 months, then at least monthly for an additional 8 months, coaches were allowed to contact their clients more frequently. Contacts were made before each primary care visit to plan for the encounter, including asking questions and encouraging the participant to reach back out to the office if needed. Contacts were largely unstructured and highly individualized to focus on the goals selected by the participants. Contacts were documented on forms designed to facilitate interactions while tracking intervention fidelity over the 10 -month intervention period. Intervention fidelity was also monitored through weekly contacts with peer coaches and a random selection of intervention participants and through weekly review of contact forms.

Participants in both study arms received a 1-hour group diabetes education class at enrollment covering diabetes basics, healthy eating, stress reduction, physical activity, social support, and how to get the most out of doctor visits. They also received a 5 -minute counseling session and a diabetes report card showing their own baseline glycated hemoglobin $\left(\mathrm{HbA}_{1 \mathrm{c}}\right), \mathrm{BP}$, low-density lipoprotein cholesterol (LDL-C) and body weight.

\section{Study design}

The clusters in this cluster-randomized trial were communities, blocked on smaller vs larger community size, with participants nested within communities (Appendix Table 1). As detailed in an earlier report, ${ }_{1}^{17}$ we initially recruited primary care practices to provide patients, but we eventually expanded recruitment into surrounding communities to meet recruitment targets. A cluster-randomized design was used to minimize contamination, but the intervention acted at the individual level, which we therefore considered the appropriate level for analysis. The study statistician used a random-number generator to assign clusters to the two trial arms. The nature of the study precluded blinding participants and peer coaches to trial arm assignment. The study's primary outcomes were changes in $\mathrm{HbA}_{1 \mathrm{c}}$, systolic $\mathrm{BP}, \mathrm{LDL}-\mathrm{C}$, body mass index (BMI), and quality of life..$^{21,22}$ Diabetes distress 
and patient activation were secondary outcomes. ${ }^{23-25}$ The trial was designed to provide $80 \%$ power to detect clinically important differences in $\mathrm{HbA}_{\mathrm{cc}}$ $(0.4 \%)$, systolic blood pressure $(4 \mathrm{~mm} \mathrm{Hg})$ and LDL-C (6 mg/dL). The sample size calculations included a variance inflation factor to account for the clusterrandomized design and $20 \%$ attrition.

\section{Analysis plans}

Characteristics including age, race, sex, education, annual household income, total number of prescription medications, and use of insulin were contrasted by study arm. Sociodemographic differences between study arms with $P$ values less than .10 were included in the models. We also included a variable reflecting the season of the year, since $\mathrm{HbA}_{1 \mathrm{c}}$ is known to vary by season. ${ }^{26}$ Because reach was of major interest, we completed follow-up on as many participants as possible at the expense of extending follow-up beyond the goal of 1 year. Therefore, we included a term in the models reflecting the number of days between baseline and follow-up. In a secondary analysis, we also included the number of contacts in models to examine the role of intervention dose. All modeling was done using the $\mathrm{R}$ statistical programming language version 3.0.128; additive models were fit using the mixed GAM computation vehicle (mgcv) package ${ }^{29}$ and smoothed plots were generated using the ggplot2 package. ${ }^{30}$ All analyses were intention-to-treat, regardless of intervention dose.

Following the study's analytic plan, the initial analysis tested for a difference in the change in study outcomes between intervention and control group participants with follow-up no more than 15 months after baseline, adjusting for clustering (the unit of randomization) and for baseline imbalance in sociodemographic characteristics between intervention and control groups. We also conducted analyses including participants who were followed up more than 15 months after baseline using Generalized Additive Mixed Models (GAMM) because of nonlinear effects. ${ }^{27}$ The estimate of the degrees of freedom (EDF) required of these models served as an indicator of nonlinearity.
Sensitivity analyses considered interactions between baseline values and elapsed time from baseline to follow-up, yielding similar results. Because of possible bias due to associations between participant characteristics and time to follow-up, we also examined outcome and baseline characteristics separately by whether elapsed time from baseline to follow-up was greater or less than 15 months.

\section{RESULTS}

Of 424 participants enrolled, 360 completed follow-up ( $84.9 \%$ retention) (Figure 1). There were no significant differences at baseline in sociodemographics or baseline measures of the study outcomes between those with and without follow-up (Appendix Table 2). Those without follow-up tended to be younger and to have more depressive symptoms.

The characteristics of the 360 participants who completed the study are shown in Table 1 . Their mean age was 60.2 years, $75.3 \%$ were women, $87.4 \%$ were African American, and nearly three-fourths had a high school education or less. We observed significant differences between study arms in race, education, and baseline quality of life.
Figure 1. Consort diagram.

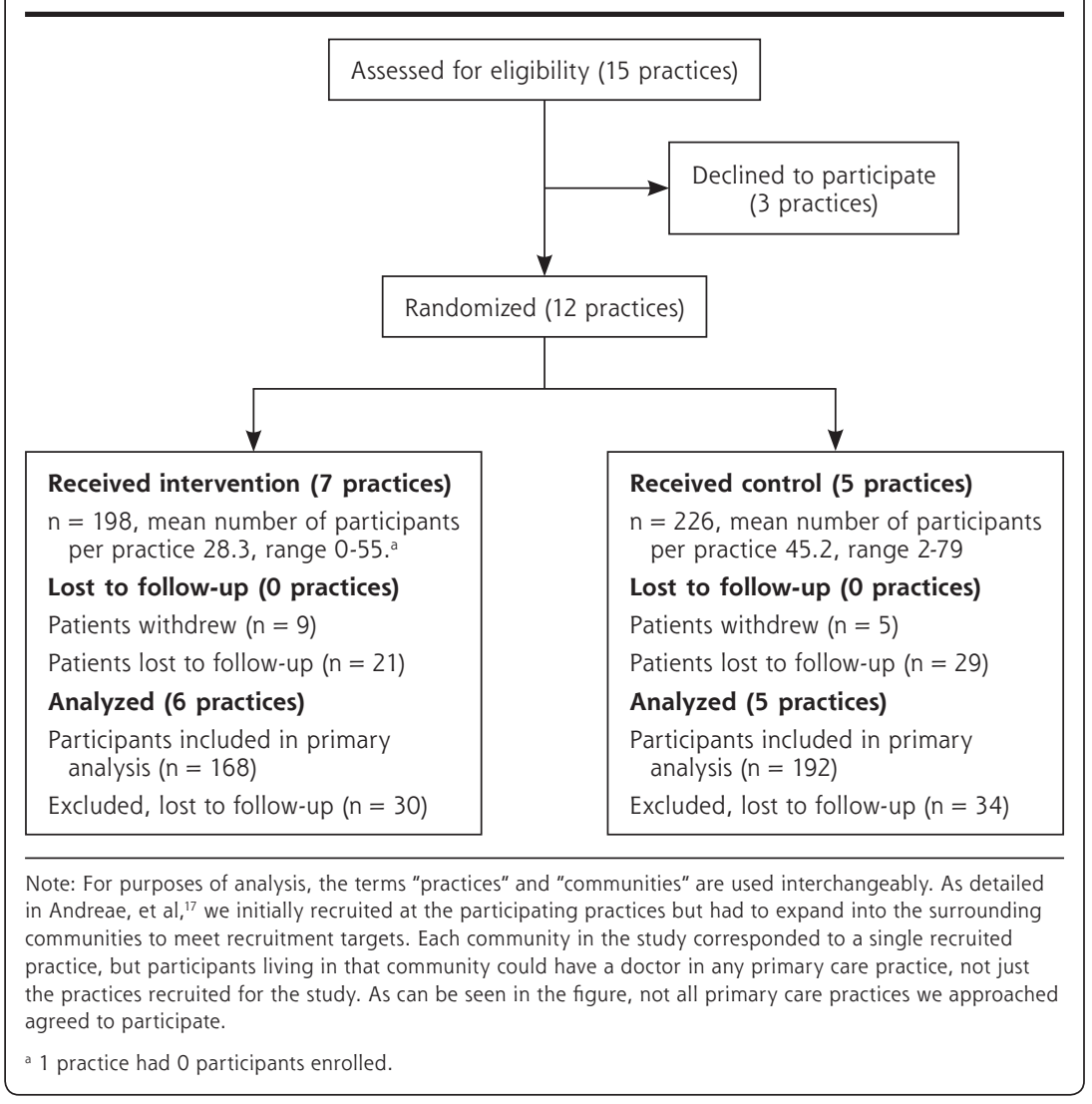


Table 1. Characteristics of Participants in the Cluster Randomized Trial

\begin{tabular}{|c|c|c|c|c|}
\hline & $\begin{array}{c}\text { All } \\
(\mathrm{N}=360)^{\mathrm{a}}\end{array}$ & $\begin{array}{c}\text { Control } \\
(n=192)\end{array}$ & $\begin{array}{l}\text { Intervention } \\
(n=168)\end{array}$ & $\begin{array}{c}P \\
\text { value }^{b}\end{array}$ \\
\hline Age, mean (SD), y & $60.2(12.1)$ & $61.1(12.4)$ & $59.2(11.8)$ & .14 \\
\hline Female, No. (\%) & $271(75.3)$ & $140(72.9)$ & $131(78.0)$ & .32 \\
\hline Blacks, No. (\%) & $313(87.4)$ & $155(81.2)$ & $158(94.6)$ & $<.001$ \\
\hline \multicolumn{5}{|l|}{ Education, No. (\%) } \\
\hline$\leq$ High school & $111(31.2)$ & $58(30.5)$ & $53(31.9)$ & \multirow[t]{3}{*}{.05} \\
\hline High school graduate & $151(42.4)$ & $72(37.9)$ & $79(47.6)$ & \\
\hline Some college, college graduate & $94(26.4)$ & $60(31.6)$ & $34(20.5)$ & \\
\hline \multicolumn{5}{|l|}{ Annual household income, No. (\%) } \\
\hline$\geq \$ 40,000$ & $32(8.9)$ & $22(11.5)$ & $10(6.0)$ & \multirow[t]{3}{*}{.10} \\
\hline$<\$ 40,000$ & $293(81.4)$ & $155(80.7)$ & $138(82.1)$ & \\
\hline Declined to report & $35(9.7)$ & $15(7.8)$ & $20(11.9)$ & \\
\hline Number of medications, mean (SD) & $7.7(4.0$ & $7.9(4.0$ & $7.5(4.0$ & .26 \\
\hline Adherent to medications, No. $(\%)^{c}$ & $189(52.5)$ & $106(55.2)$ & $83(49.4)$ & .33 \\
\hline Treated with insulin, No. (\%) & $142(39.4)$ & $75(39.1)$ & 67 (39.9) & .99 \\
\hline Baseline hemoglobin $A_{1 c}$, mean (SD), \% & $7.9(2.0)$ & $7.9(1.9)$ & $8.0(2.1)$ & .56 \\
\hline Baseline body mass index, mean (SD), kg/m² & $36.3(8.5)$ & $36.0(9.1)$ & $36.5(7.7)$ & .57 \\
\hline Baseline systolic blood pressure, mean (SD), mm Hg & $135.2(21.4)$ & $135.8(21.2)$ & $134.6(21.7)$ & .61 \\
\hline Baseline LDL-C, mean (SD), mg/dL & $110.6(38.2)$ & $111.6(38.8)$ & $109.4(37.6)$ & .59 \\
\hline Baseline quality of life score, mean (SD) ${ }^{d}$ & $0.76(0.2)$ & $0.78(0.2)$ & $0.74(0.2)$ & .03 \\
\hline Baseline diabetes distress score, ${ }^{*}$ mean (SD)e & $2.1(1.2)$ & $2.1(1.2)$ & $2.1(1.2)$ & .92 \\
\hline Baseline activation score, ${ }^{*}$ mean $(\mathrm{SD})^{f}$ & $27.8(6.1)$ & $28.0(6.7)$ & $27.5(5.3)$ & .51 \\
\hline \multicolumn{5}{|c|}{ LDL-C = low density lipoprotein cholesterol; SD = standard deviation. } \\
\hline \multicolumn{5}{|c|}{$\begin{array}{l}\text { a Includes all participants who provided both baseline and follow-up data. } \\
\text { b P-values were derived from } x^{2} \text { tests for categorical variables and } t \text {-tests for continuous variables. } \\
\text { c We assessed medication adherence using the 4-item Morisky scale (dichotomized as adherent or not). } \\
\text { d We assessed quality of life using the EuroQol 5D (range 0-1.0). } \\
\text { e We assessed diabetes distress using the Diabetes Distress Scale (range 1-6, with scores } \geq 3 \text { indicative of moderate or greater distress). } \\
\text { 'We assessed activation using the Patient Activation Measure (range 13-57). }\end{array}$} \\
\hline
\end{tabular}

Among the 168 participants in the intervention arm, the total number of reported contacts over 10 months ranged from 0 to $58 ; 54$ (32.1\%) had 17 or more contacts (the number specified in the protocol), $115(68.5 \%)$ had 10 or more contacts, and $14(8.3 \%)$ had no contacts; the mean number of contacts was 13.3 (SD 8.1). The main reason some participants had fewer contacts than anticipated was that their telephone numbers were disconnected. The number of contacts was not consistently associated with outcomes (Appendix Table 3). To better understand how the intervention could have exerted favorable effects without any association with the frequency, timing, or total number of contacts, we debriefed the peer advisors and community coordinators. In many cases, peer advisors already knew their clients and had more frequent contacts than the formal contacts that were documented as part of the study. For example, many saw each other at school events, at church on Sundays, or during usual daily activities within the community. If a peer advisor saw a client during these casual contacts, he or she almost always sought out the client (and vice versa) and discussed the intervention and the client's goal, providing support outside the schedule of contacts; we encouraged documentation of these contacts, but peer advisors reported that it was difficult to remember to do so. Therefore, the total number of formal intervention contacts may not reflect the total intervention dose in many cases. Furthermore, peer advisors reported that many continued to keep in touch with their clients well after the official intervention period especially with the prolonged follow-up data collection period, this makes it difficult to estimate intervention dose using formally collected contact frequency.

All but 1 intervention participant reported high satisfaction with the program, with $67 \%$ reporting the highest level of satisfaction. Among participants in the intervention arm, 93\% chose to work on weight loss and exercise, $19 \%$ on stress reduction, $9 \%$ on medication adherence, $3 \%$ on improving physician visits, and $11 \%$ on other goals.

The changes in study outcomes between study arms were limited in those followed up within 15 months, adjusted only for clustering and education, which was imbalanced at baseline; race was not included because there were too few whites (Table 2). 
The only significant difference was change in activation, which increased 2.64 points in the intervention group vs 0.69 in the control group $(P=0.04)$.

Figure 2 and Figure 3 present two views of each of the study's primary and secondary outcomes, respectively:

- Smoothed plots of the raw change scores, by time from baseline to follow-up (the top panel of each pair)

- The difference in change by time from baseline to follow-up attributable to the intervention in GAMMs adjusted for time interval between baseline and follow-up, season, baseline value, clustering, and education (the bottom panel of each pair).

The 2 vertical lines mark 12 and 15 months after baseline.

For each outcome, a $P$ value less than .05 indicates a statistically significant intervention effect. When the overall term is statistically significant, the EDF indicate the degree of nonlinearity required to model the intervention change over time, with larger values indicating greater nonlinearity. Note that although significance indicates that the overall trajectories of change over the time interval differed between study groups, it does not indicate whether the difference was statistically significant at any particular time. ${ }^{27}$

Figure 2A depicts changes in $\mathrm{HbA}_{1 \mathrm{c}}$, revealing no statistically significant intervention effect. Figure $2 \mathrm{~B}$ shows a significant time trend for systolic BP with a larger reduction in the intervention group seen primarily after 15 months. Figure $2 \mathrm{C}$ depicts a rise over time in LDL-C, with no significant intervention effects. The top panel in Figure 2D shows little indication of a difference between groups in the raw BMI data; the

Table 2. Mean Changes in Primary Study Outcomes from Baseline to Follow-up for Participants Followed Up Within 15 Months, by Treatment Arm

\begin{tabular}{|c|c|c|c|}
\hline Outcome & $\begin{array}{l}\text { Intervention } \\
(n=138)\end{array}$ & $\begin{array}{c}\text { Control } \\
(n=130)\end{array}$ & $\begin{array}{c}P \\
\text { value }^{a}\end{array}$ \\
\hline Hemoglobin $A_{1 c}$ mean (SD), \% & $-0.004(1.5)$ & $0.070(1.3)$ & .68 \\
\hline Body mass index, mean (SD), $\mathrm{kg} / \mathrm{m}^{2}$ & $-0.23(2.4)$ & $-0.49(2.8)$ & .44 \\
\hline Systolic blood pressure, mean (SD), $\mathrm{mm} \mathrm{Hg}$ & $-0.41(21.3)$ & $-1.88(22.8)$ & .59 \\
\hline $\mathrm{LDL}-\mathrm{C}$, mean (SD), mg/dL & $3.40(31.5)$ & $-0.18(31.3)$ & .35 \\
\hline Quality of life score, mean (SD) ${ }^{\mathrm{b}}$ & $-0.006(0.2)$ & $-0.017(0.2)$ & .63 \\
\hline Diabetes distress score, mean $(S D)^{c}$ & $-0.13(1.2)$ & $-0.29(1.1)$ & .26 \\
\hline Activation score, mean (SD) ${ }^{\mathrm{d}}$ & $2.64(7.4)$ & $0.69(7.0)$ & .04 \\
\hline \multicolumn{4}{|c|}{ LDL-C = low density lipoprotein cholesterol; SD = standard deviation. } \\
\hline \multicolumn{4}{|c|}{$\begin{array}{l}\text { a } P \text { values are for intervention compared with control group mean values, adjusted for clustering and for } \\
\text { education, which was imbalanced at baseline across treatment arms. P-values were derived from } \chi^{2} \text { tests } f \\
\text { categorical variables and } t \text {-tests for continuous variables. } \\
\text { b We assessed quality of life using the EuroQol 5D (range 0-1.0). } \\
\text { "We assessed diabetes distress using the Diabetes Distress Scale (range 1-6 with scores } \geq 3 \text { indicative of } \\
\text { moderate or greater distress). } \\
{ }^{d} \text { We assessed activation using the Patient Activation Measure (range 13-57). }\end{array}$} \\
\hline
\end{tabular}

bottom panel shows a significant, highly nonlinear difference between groups in the adjusted model. Intervention group participants followed up earlier and later than 15 months experienced greater weight loss than control participants followed at the same times, but those followed around 15 months may have had slight weight gain compared with control participants. Figure $2 \mathrm{E}$ shows that change in quality of life varied over time, with lowering of quality of life for intervention group participants compared with controls followed up at about 15 months, but improved quality of life for intervention participants assessed later, with an overall statistically significant difference between groups.

The intervention effect for diabetes distress varied nonlinearly over time (Figure $3 \mathrm{~A}$ ), most noticeably in a relative increase of distress in the intervention group around 15 months. A relative increase in activation among the intervention group was found early on, apparently peaking at around 14 months (Figure 3B). The differences in trajectory were statistically significant in both cases.

Of the 360 participants, $270(75 \%)$ were followed up within 15 months, and they are contrasted with those followed up later, by trial arm, in Appendix Table 4. The 131 members of the control group followed up within 15 months (68\% of all control group participants) were older and tended to be on more medications, with lower baseline $\mathrm{HbA}_{1 \mathrm{c}}$ but higher baseline LDL-C compared with those followed up after 15 months. The 139 members of the intervention group followed up within 15 months (83\% of all intervention group participants) tended to be on more medications and to have lower systolic BP and higher activation scores at baseline. Differences between the two groups were not statistically significantly for the rest of the characteristics tracked. Because of the differences described above, we conducted additional analyses incorporating interactions between baseline characteristics and time to follow-up in adjusted models yielding similar findings, suggesting that differences in change in outcomes over time were unlikely to be attributable to differences between patients followed up within 15 months and those followed up later.

\section{DISCUSSION}

This telephone-delivered peercoaching intervention activated 
Figure 2 A-E. Control-intervention arm differences in change in primary outcome measures for all study participants, showing raw change scores (top of each panel) and change scores from generalized additive models adjusting for differences in time from baseline to follow-up, season, baseline value, clustering, education, and race.
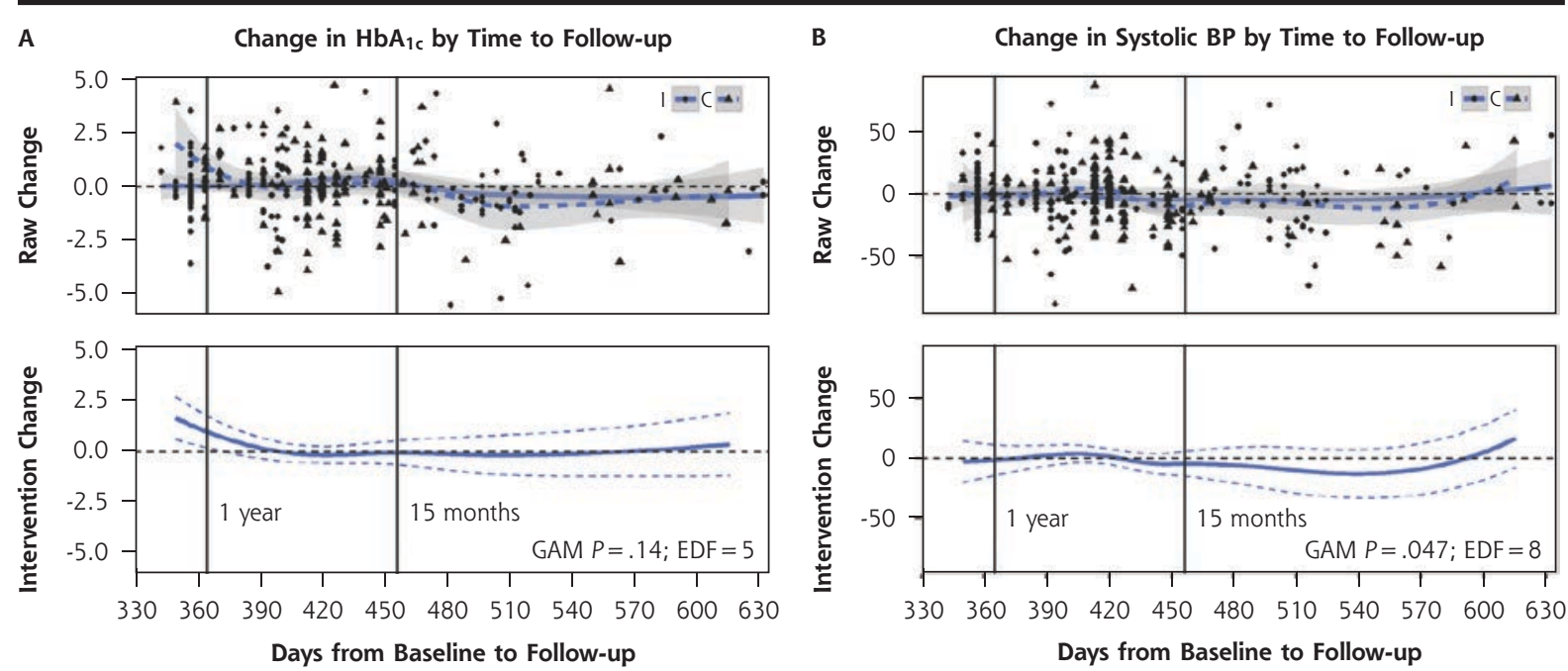

C Change in LDL-C by Time to Follow-up

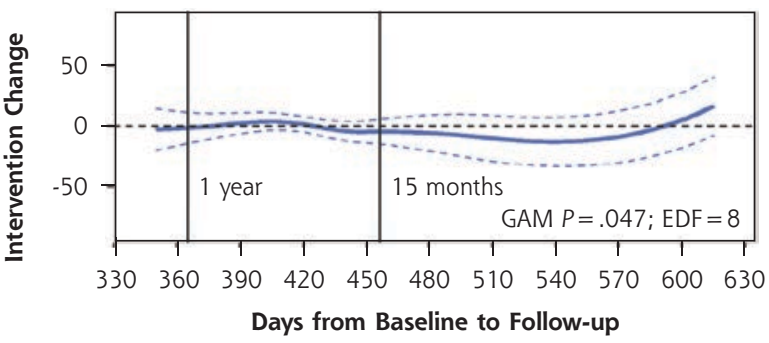

D
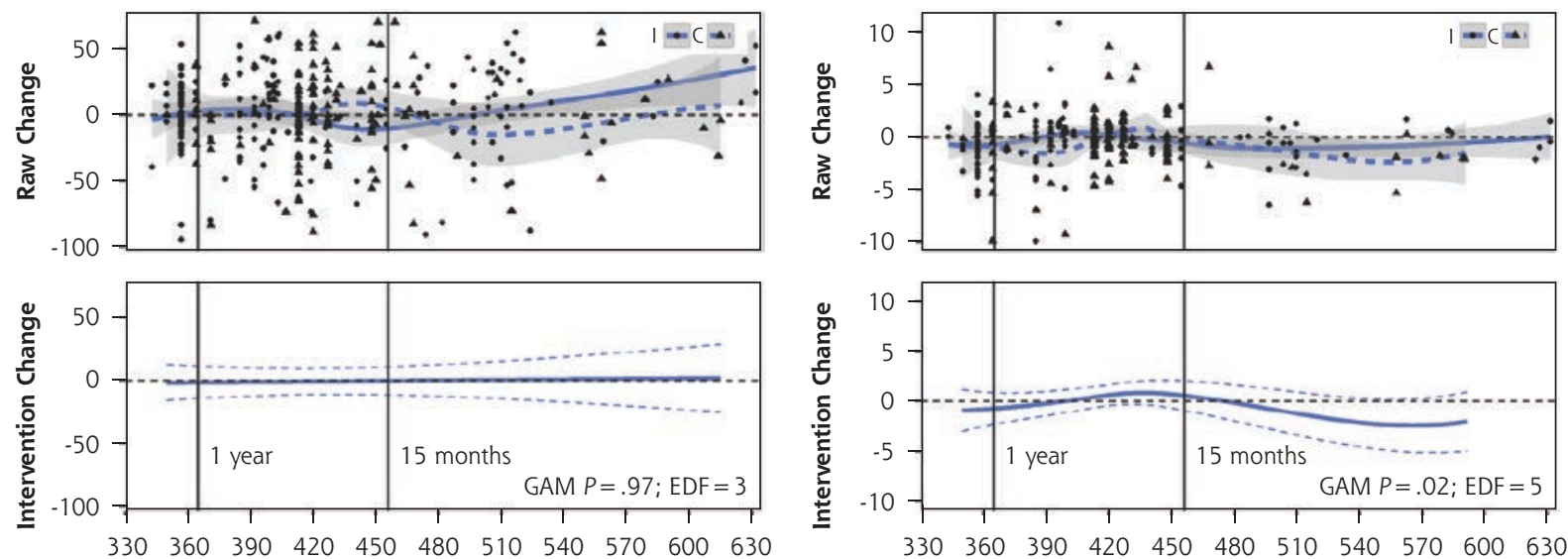

Days from Baseline to Follow-up

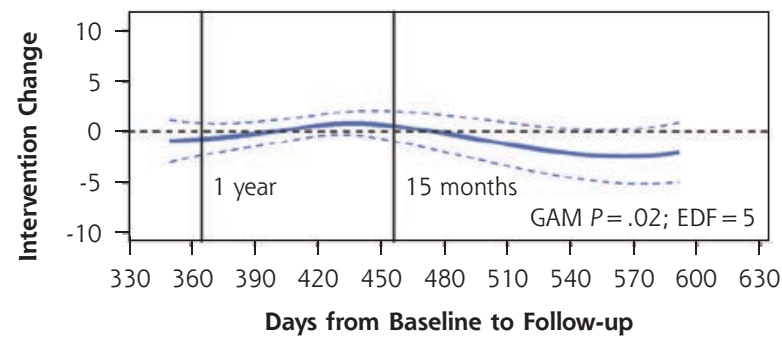

E Change in Quality of Life by Time to Follow-up

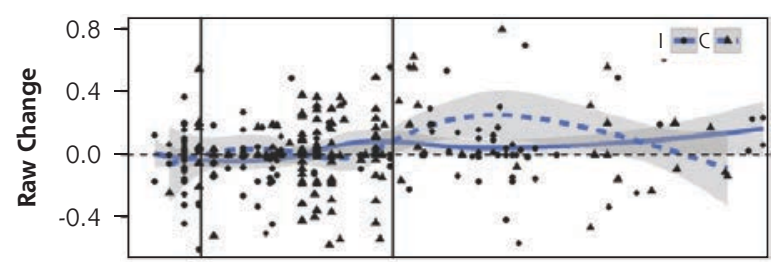

Note: In each panel, the top graph presents the raw change scores for each participant, with circles and solid lines signifying control arm and triangles and dotted lines signifying intervention arm. The $\mathrm{x}$ axis shows the time in days between baseline and follow-up. Vertical lines show the 12 and 15-month follow-up points. The bottom graph in each panel presents the differences between intervention and control change scores from generalized additive mixed models with $p$-values from tests of statistical significance of the difference between control and intervention arms. See the article text as well.

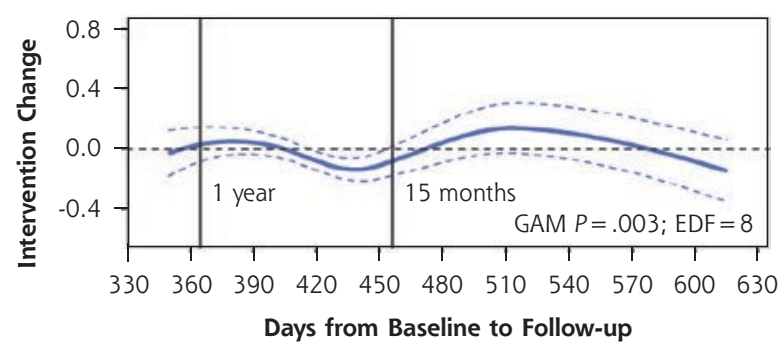




\begin{abstract}
Figure $3 \mathrm{~A}$ and B. Control-intervention arm differences in change in secondary outcome measures for all study participants by study arm showing raw change scores (top of each panel) and change scores from generalized additive models adjusting for differences in time from baseline to follow-up, season, baseline value, clustering, education, and race.
\end{abstract}

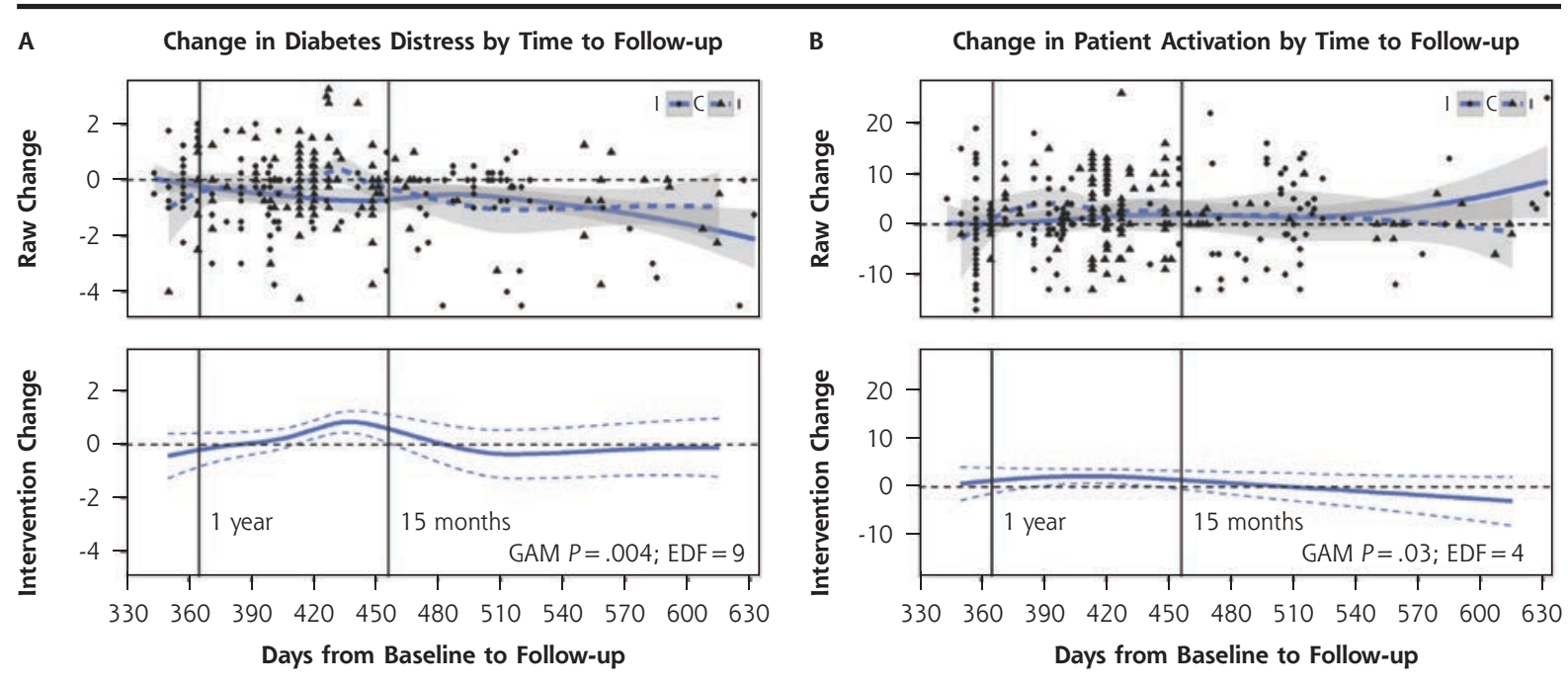

$\mathrm{EDF}=$ estimated degrees of freedom; GAMM = generalized additive mixed models.

In each panel, the top graph presents the raw change scores for each participant with circles and solid lines signifying control arm and triangles and dotted lines signifying intervention arm. The $x$ axis shows the time in days between baseline and follow-up. Vertical lines show the 12 and 15 -month follow-up points. The bottom graph in each panel presents the differences between intervention and control change scores from generalized additive models with $p$-values from tests of statistical significance between control and intervention arms. See the article text as well.

participants but had no other significant effects on the study's outcomes when analysis was restricted to those followed up within 15 months, a timeframe commonly used for 1-year follow-up studies. An alternative analytic approach, however, included all participants who were followed up, thereby extending reach and generalizability, and demonstrated a significant effect of peer coaching on systolic BP, BMI, and quality of life, but not on $\mathrm{HbA}_{1 \mathrm{c}}$ or LDL-C. These effects varied over time, extending well into the second year after the official end of the intervention. We found little evidence of differences between participants followed up earlier and later than 15 months that could explain these findings, and many of the volunteer peer coaches continued unmeasured contacts with clients beyond the trial period, strengthening the view that the differences represent an intervention effect.

This study provides an instructive example of the benefits and disadvantages of extending follow-up to maximize retention. In addition to improving generalizability, a major emphasis in real-world studies, the prolonged follow-up allowed us to model the trajectory of the intervention effect over time, providing more information than a simple cross-section of differences at 1 year. At the same time, though, it complicated the interpretation, since GAMMs do not provide information about effectiveness at any given point in time.
Given that this was a pragmatic trial in communities with very real barriers to health care access and study participation, the approach shown here provides a way to use all available data to construct a more nuanced picture of the intervention than the more traditional approach, which would lead to the conclusion that the intervention had no effect on the targeted outcomes. We note that certain outcomes tracked together. For instance, the slight weight gain in participants followed up at 15 months was paralleled by a slight decrement in quality of life, and improved BP and quality of life paralleled the more pronounced weight loss observed in those who were followed up later. An effect of peer support on weight loss is plausible, given the large number of participants selecting diet and exercise as their personal goals. Likewise, patient activation, which was greatest relatively early in follow-up, would be expected to be an early change that is followed weeks later by improvements such as weight loss.

We observed a disturbing increase in diabetes distress at about 15 months among intervention participants. This finding suggests that more emphasis on emotional support may be needed in interventions similar to this one.

Our findings complement several recent reports demonstrating benefits of peer support in diabetes. A Detroit study found beneficial effects of a community health worker intervention on $\mathrm{HbA}_{1 \mathrm{c}}$ but not weight, 
cholesterol, or BP. ${ }^{31}$ Another study reported improved $\mathrm{HbA}_{\mathrm{lc}}$ with peer support compared with nurse care management in the Veterans Administration. ${ }^{12}$ A peer support intervention based in public health clinics in San Francisco demonstrated improved $\mathrm{HbA}_{1 \mathrm{c}}$ at 6 months, $30 \%$ of participants were African Americans. ${ }^{13}$ Our study participants had relatively low baseline $\mathrm{HbA}_{\mathrm{cc}}$ levels, with most participants choosing to work on diet and exercise, possibly explaining the lack of impact on $\mathrm{HbA}_{1 \mathrm{c}}$ and favorable effects on weight loss, $\mathrm{BP}$, and quality of life.

This study's limitations include limited power for the analysis of temporal trends. Unmeasured characteristics could have differed between those followed earlier and those followed up later, contributing to the findings. Use of respondent-driven sampling for recruitment of participants and a convenience sample for recruitment of communities did not assure a representative sample of diabetic residents, but our inclusion in the analysis of all participants who were followed up improved generalizability. The study was not able to be blinded, we were not able to objectively assess differences in coaching style, which could impact the intervention's effects, and we did not have information available about the nature and frequency of continued contacts beyond the 10 -month intervention period.

In conclusion, in an underserved, hard-to-reach, rural region populated primarily by African Americans, a brief education session plus an unstructured peer coaching intervention delivered over the telephone by community volunteers to residents with diabetes had time-dependent and variable effects on BP, BMI, quality of life, and patient activation, relative to brief education alone.

To read or post commentaries in response to this article, see it online at http://www.annfammed.org/content/13/Suppl_1/S18.

Submitted July 25, 2014; submitted, revised, March 2, 2015; accepted March 12, 2015.

Key words: Peer health coaching; self-management support; diabetes; primary care

Funding support: Funding for this research was provided by the American Academy of Family Physicians Foundation through the Peers for Progress program with support from the Eli Lilly and Company Foundation.

Supplementary materials: Available at http://www.AnnFamMed. org/content/13/Suppl_1/S18/suppl/DC1/

\section{REFERENCES}

1. Roger VL, Go AS, Lloyd-Jones DM, et al; American Heart Association Statistics Committee and Stroke Statistics Subcommittee. Heart disease and stroke statistics-2012 update: a report from the American Heart Association. Circulation. 2012;125(1):e2-e220.
2. Tullos A. The Black Belt. Southern Spaces. 2004. http://www.southernspaces.org/2004/black-belt. Published Apr 19, 2004. Accessed Apr 22, 2015.

3. State $\varepsilon$ County Quick Facts. 2014. http://quickfacts.census.gov/qfd/ states/01000.html. Accessed Dec 1, 2014.

4. Alabama Poverty Rate by County. 2014. http://www.indexmundi. com/facts/united-states/quick-facts/alabama/percent-of-people-of-allages-in-poverty\#map. Accessed Dec 1, 2014.

5. Primary Care Physicians by Field. State Health Facts. 2014. http://kff. org/other/stateindicator/primary-care-physicians-by-field/. Accessed Dec 1, 2014.

6. Board of Medical Examiners. 2014. http://www.albme.org/. Accessed Dec 1, 2014.

7. State Report Cards. Quality Counts. 2014. http://www.edweek.org/ ew/qc/2014/state_report_cards.html. Accessed Dec 8, 2014.

8. Curran WJ. The tuskegee syphilis study. N Engl J Med. 1973;289 (14):730-731.

9. Viswanathan M, Kraschnewski J, Nishikawa B, et al. Evidence report/ technology assessment, no.181: Outcomes of community health worker interventions. AHRQ publication no. 09-E014; June 2009.

10. Heisler M. Different models to mobilize peer support to improve diabetes self-management and clinical outcomes: evidence, logistics, evaluation considerations and needs for future research. Fam Pract. 2009;(Mar):17.

11. Heisler M, Spencer M, Forman J, et al. Participants' assessments of the effects of a community health worker intervention on their diabetes self-management and interactions with healthcare providers. Am J Prev Med. 2009;37(6)(Suppl 1):S270-S279.

12. Heisler M, Vijan S, Makki F, Piette JD. Diabetes control with reciprocal peer support versus nurse care management: a randomized trial. Ann Intern Med. 2010;153(8):507-515.

13. Thom DH, Ghorob A, Hessler D, De Vore D, Chen E, Bodenheimer TA. Impact of peer health coaching on glycemic control in lowincome patients with diabetes: a randomized controlled trial. Ann Fam Med. 2013;11(2):137-144.

14. World Health Organization. Peer Support Programmes in Diabetes: Report of a WHO Consultation, 5-7 November 2007. Geneva, Switzerland: WHO, 2008.

15. Fisher EB, Boothroyd RI, Coufal MM, et al. Peer support for selfmanagement of diabetes improved outcomes in international settings. Health Aff (Millwood). 2012;31(1):130-139.

16. Fisher EB, Earp JA, Maman S, Zolotor A. Cross-cultural and international adaptation of peer support for diabetes management. Fam Pract. 2010;27(Suppl 1):i6-i16.

17. Andreae SJ, Halanych JH, Cherrington A, Safford MM. Recruitment of a rural, southern, predominantly African-American population into a diabetes self-management trial. Contemp Clin Trials. 2012;33 (3):499-506.

18. Cherrington A, Martin MY, Hayes M, et al. Intervention mapping as a guide for the development of a diabetes peer support intervention in rural Alabama. Prev Chronic Dis. 2012;9:E36.

19. Lewis MW, Cherrington AL, Gamboa CM, Halanych JH, Martin MY, Safford MM. Assessing peer advisor intervention fidelity using video skits in a peer support implementation trial. Health Promot Pract. 2014;15(5):759-767.

20. Heckathorn DD. Respondent-driven sampling: A new approach to the study of hidden populations. Soc Probl. 1997;44(2):174-199.

21. Rabin R, de Charro F. EQ-5D: a measure of health status from the EuroQol Group. Ann Med. 2001;33(5):337-343.

22. Kind P, Brooks R, Rabin R. EQ-5D Concepts and Methods: a Developmental History. Berlin: Springer Verlag; 2005.

23. Polonsky WH, Fisher L, Earles J, et al. Assessing psychosocial distress in diabetes: development of the diabetes distress scale. Diabetes Care. 2005;28(3):626-631. 
24. Hibbard JH, Stockard J, Mahoney ER, Tusler M. Development of the Patient Activation Measure (PAM): conceptualizing and measuring activation in patients and consumers. Health Serv Res. 2004;39 (4 Pt 1):1005-1026.

25. Hibbard JH, Mahoney ER, Stockard J, Tusler M. Development and testing of a short form of the patient activation measure. Health Serv Res. 2005;40(6 Pt 1):1918-1930.

26. Tseng CL, Brimacombe $M$, Xie $M$, et al. Seasonal patterns in monthly hemoglobin A1c values. Am J Epidemiol. 2005;161(6):565-574.

27. Richman J, Andreae S, Safford MM. Challenges of prolonged follow-up and temporal imbalance in pragmatic trials: Analysis of the ENCOURAGE Trial. Ann Fam Med. 2015;13(Suppl_1):S66-S72.
28. R: A language and environment for statistical computing 2013; http://www.Rproject.org/. Accessed May 27, 2014.

29. Wood SN. Fast stable restricted maximum likelihood and marginal likelihood estimation of semiparametric generalized linear models. J R Stat Soc, B. 2011;73(1):3-36.

30. Wickham H. ggplot2: Elegant graphics for data analysis. New York: Springer; 2009.

31. Spencer MS, Rosland AM, Kieffer EC, et al. Effectiveness of a community health worker intervention among African American and Latino adults with type 2 diabetes: a randomized controlled trial. Am J Public Health. 2011;101(12):2253-2260. 